\title{
Formación por competencias y acreditación de calidad: su convergencia en el curriculum*
}

\section{Competency-based training and accreditation of quality: its convergence in the curriculum \\ Formação por competências $\epsilon$ certificação de qualidade: sua convergência no curriculum}

Ángela María Restrepo Jaramillo**

Fundación Universitaria Luis Amigó, Medellín, Colombia

Resumen. Los procesos de autoevaluación con fines de acreditación y la formulación de competencias en el marco de los programas de pregrado requieren mirarse en y desde su relación con el currículo; no obstante la práctica devela que este vínculo aún no se teje; por ello es fundamental una reflexión a la luz de la revisión crítica de literatura sobre el tema que permita entender sus nexos a partir de las consideraciones de un enfoque práctico.

Palabras clave. Plan de estudios, currículo, educación superior, autoevaluación, acreditación, calidad, competencias (Tesauro Unesco).

Artículo de revisión de tema que indaga sobre la relación entre los procesos de autoevaluación con fines de acreditación y el componente curricular en los programas de pregrado.

** Magíster en Educación y Desarrollo Humano, Especialista en Educación. Docente ocasional de la Universidad San Buenaventura Medellín y docente auxiliar de la Fundación Universitaria Luis Amigó, Medellín, Colombia. E-mail: angelarepo@ hotmail.com; angela.restrepoja@amigo.edu.co 
Abstract. The self-regulation processes required for accreditation and competence development an undergraduate program entails an analysis of the relation with the curriculum; however, practice has clearly shown that such connection is yet to be established. Such situation brings us to the need of a literature review to reflect upon this topic in order to shed some light on such association based on the considerations of a practical approach.

Keywords. Curriculum, superior education, self-regulation processes, accreditation, competence development (Thesaurus Unesco).

Resumo. Os processos de autoavaliação com finalidade de certificação e a formulação de competência na fronteira dos programas de graduação requerem uma mirada em e a partir de sua relação com o currículo; entretanto, a prática revela que esse vínculo ainda não se mescla; por isso é fundamental uma reflexão à luz da revisão crítica de literatura sobre o tema que permita entender seus nexos a partir das considerações de um enfoque prático.

Palavras chave. Plano de estudos, currículo, educação superior, auto avaliação, certificação, qualidade, concorrência (Tesaurus Unesco).

\section{Introducción}

Después de todas las implicaciones que tuvo la presión y simultáneamente la necesidad de hablar de una formación por competencias y de las dinámicas implícitas y explícitas que trae consigo, particularmente el proceso de autoevaluación con miras a la acreditación de los programas; y como derivación de las actividades, productos, desaciertos, logros y decisiones emanados de los mismos, surge la necesidad de una profunda y obligada reflexión sobre el curriculum, como un tema en el que ambos (competencias y procesos de acreditación) convergen y que coincidencialmente parece haberse olvidado o desconocido, y esa distracción aparente o esta ignorancia sintomática pueden entenderse en la medida en que se busca definir el curriculum, desde un enfoque específico, para posteriormente redimensionarlo y resignificarlo.

En consecuencia es pertinente iniciar un recorrido por dos de sus conceptualizaciones para dilucidar algunos detalles que más allá de situar en la historia de las prácticas educativas formales y escolarizadas, 
abren la posibilidad de caracterizar los intereses rectores en los procesos de transmisión de conocimientos, en los de enseñanza aprendizaje o en los de formación en general.

\section{Urgencia de una educación en competencias}

Desde el año 1998 la Conferencia Mundial sobre la Educación Superior llevada a cabo en París (Unesco, 1998, p. 5), se formalizó la urgencia de una educación en competencias que permitiese «superar el mero dominio cognitivo de las disciplinas» para promover asimismo, el conocimiento práctico, el trabajo en equipo, la capacidad de análisis en contextos multiculturales caracterizados por el auge de nuevos productos científicos y tecnológicos.

A partir de ese momento múltiples y muy variados requerimientos de carácter nacional, internacional y global, han exigido a las universidades el fortalecimiento de la calidad de la educación en la perspectiva de formar profesionales con «competencias sólidas para el mundo de hoy y de mañana» (Unesco, 2009, p. 2).

Para el cumplimiento de esa tarea, un sinnúmero de planes de estudio, de programas de pregrado básicamente, fueron expuestos a revisión con el interés de ajustarlos o renovarlos; así su reformulación trazada desde la Conferencia Mundial del 98 se hizo paulatinamente una realidad cuya evidencia se plasmó en documentos en los cuales se vislumbraban como novedad la definición de competencias con diversas denominaciones ${ }^{1}$ como por ejemplo las específicas y las genéricas (Tobón, Rial, Carretero, y García, 2006, p. 106) básicas, genéricas, específicas y/o laborales (Proyecto Tuning, 2007, p. 37), básicas, genéricas y especializadas (Cabrera y González, 2006, p. 9).

En consecuencia uno de los productos más explícitos fue la escritura de tales competencias que se erigieron en el marco de referencia de los perfiles de formación para demostrar, tal vez sólo desde la «forma», que los programas estaban «in» en cuanto a exigencias externas.

1 Las categorizaciones de competencias más aceptadas en la actualidad son según Cabrera y González (2006) las básicas, las genéricas, las especializadas o específicas, las laborales - que incluyen las profesionales y las de egreso-. 
No obstante la necesidad de atender con apremio a esta requisitoria y probablemente el desconocimiento de las implicaciones y la envergadura de dicho propósito, no permitieron adentrarse en el fondo de lo que implicaba ya que el desafío más allá de la enunciación y de la instauración de cambios de carácter instrumental, exigía tácitamente una mirada sistemática e integral al curriculum.

En consecuencia la toma de decisiones resultante fue nula o restringida, tal vez incluso, por la misma tendencia a pensarlo exclusivamente como un plan de estudios o un elemento abstracto en el que a diferencia de otros «hechos concretos» como por ejemplo «el papel de los post grados, el acortamiento de los estudios" y otras reformas para el mejoramiento de la universidad (Bentonilla, Pedranzani y Clavijo, 2007, p. 26) incidieron en la instauración de cambios más deliberados.

Es incluso probable que este cometido relacionado con la formación por competencias se haya entendido solamente desde el apremio de responder únicamente a una necesidad económica en el sentido que lo plantea (Correa, 2004, pág. 18):

Tan complejo escenario que va del orden internacional al nacional y local, toca frontalmente a las políticas educativas, las cuales enfrentan la lógica económica de preparar recurso humano para el mercado de trabajo; por lo tanto, las concepciones y prácticas curriculares en los centros educativos, se mueven en la dirección de esta lógica. Sin embargo, es necesario considerar que la educación al servicio del conocimiento para la formación integral del hombre y la mujer, es asunto de mayor trascendencia, que va más allá del desarrollo de competencias para favorecer los capitales de las organizaciones productivas y de servicios (p. 18).

De ahí que el ámbito de lo educativo no puede restringirse a la «capacitación profesional» que requiere diferenciarse totalmente de la formación universitaria para no "perder el sentido de la universidad, que está para promover la autoformación de las personas, la cual es la principal de las competencias, haciendo el autoaprendizaje y el lenguaje» (Cabrera y González, 2006, p. 12).

Con el cumplimiento inmediato de la inclusión de un listado de competencias en los proyectos educativos de los programas o en los 
perfiles, probablemente sólo se optó por su estructuración para la adquisición de «aptitudes polivalentes y adaptativas como respuesta a un mundo de cambios constantes» (Iafrancesco , 2004a) pero tal vez se olvidó el tal encargo social delegado en quienes desde diversos lugares piensan la educación, de humanizarla y constituirla en el requisito sine qua non es imposible acceder a la formación integral y al desarrollo humano con todo lo que éste implica.

La diferencia fundamental entre un currículo basado en competencias y un currículo convencional, es que en el primer caso la planificación de la docencia se hace a partir de un diagnóstico prospectivo de la realidad donde se va a desempeñar el egresado... La planificación de la docencia tradicional se basa más bien en las lógicas conceptuales que especifican los especialistas del mundo académico (Cabrera y González, 2006, p. 11).

Los roles de quienes están comprometidos e involucrados con el proceso formativo cambian a la luz de esta propuesta. El estudiante y los egresados adquieren un valor definitivo: son actores que desde el análisis de la realidad contribuyen con la estructuración del marco formativo. Las prácticas de construcción de aprendizajes, de aplicación del saber, del aprender a aprender desplazan las tradicionales de enseñanza; los proceso docente educativos se dinamizan, pero conjuntamente se impactan y renuevan «diversidad de funciones y actividades universitarias» en mucho de lo que a ellas concierne.

En esta misma línea de análisis, las competencias deberán superar entonces los niveles problemáticos de la mutación de las formas verbales, es decir de su redacción, y de la «creación espontánea», para contribuir a la consolidación de propuestas curriculares muy pensadas, armónicas, articuladas como un todo en el que cada componente del proceso docente educativo cobra sentido en su diálogo con los demás, y cada decisión, vivencia y proceso institucionales se tornan legítimos por cuanto responden a un ideal de formación plasmado en la misión

Además, su ámbito conceptual y en la acción desbordará la solicitud propia del «mercado del trabajo» en la que se trata de complacer a las críticas que por tiempos se han hecho al sistema educativo por marchar en «desajuste con el sistema laboral» generando presiones que inciden en cada uno de los niveles y lograrán ser un producto legítimo 
de «la expresión de luchas y conflictos entre posiciones, perspectivas, tradiciones que devienen finalmente en un texto prescripto, con efectos regulativos sobre las prácticas de enseñanza» que recoge las intencionalidades y responsabilidades de tal sistema y sistematiza «una producción negociada sobre los modos de concebir el conocimiento legítimo y las maneras de transmitirlo» (Altermann, 2008, p. 13).

\section{Educación superior: calidad, autoevaluación y acreditación}

De manera simultánea a este tema y con un auge cada vez mayor, las cuestiones sobre la calidad de la educación se posicionaron como uno centrales en los diversos contextos económicos, políticos y académicos.

En Colombia la Ley 30 de 1992, en sus artículos 53 y 54 declaraba la creación del Sistema Nacional de Acreditación, el cual se consolidó como respuesta a la necesidad de fortalecer la calidad de la educación superior y al propósito de hacer reconocimiento público del logro de altos niveles de calidad, buscando preservar así derechos legítimos que en esta materia tienen los usuarios del sistema de educación superior y la sociedad» (CNA, 2013, p. 6).

Progresivamente las universidades se dedicaron a pensar y tomar decisiones que buscaran dicho fortalecimiento. Luego, los informes sobre las clasificaciones de las mismas -ranking- en los ámbitos nacionales como en los internacionales y las obligaciones derivadas de los procesos de renovación de registro calificado y acreditación de calidad de programas y universidades, específicamente en el contexto colombiano generaron igualmente una dinámica de revisión interna a la luz de los estándares y la estructuración de planes de acción y planes de mejoramiento que posibilitaran la consecución de las respectivas notificaciones por parte de las instancias pertinentes.

El reto que la Unesco (2009) volvía a reiterar sobre la función esencial de la «enseñanza superior contemporánea» en relación con la garantía de la calidad, se implantó, con grandes diferencias temporales para su asunción por parte de las instituciones universitarias y las universidades, con la promoción de una cultura sobre la misma, que a su vez se materializó en la implementación de sistemas de su aseguramiento y en la puesta en acción de procesos de autoevaluación que implicaban desde el ideal propugnado ese año en París, «la participación de todos los interesados» (p. 4). 
Las decisiones que mostrarían «cómo una institución y sus programas orientan su deber ser hacia un ideal de excelencia» (CNA, 2013, p. 8) se plasmaron en «aspectos a evaluar» sobre características particulares propias de ocho factores, según los Lineamientos de 2006 (CNA , 2006, p. 49) y de diez factores en los lineamientos de 2013 (CNA, 2013, p. 19).

Sobre su formulación se deduce la calidad de la educación superior alrededor del cumplimiento de tópicos referidos a: profesores con altos niveles de cualificación y con modalidades de vinculación apropiadas, la investigación, la formación integral de las personas, la pertinencia y relevancia social, los ambientes educativos más heterogéneos y flexibles, el seguimiento a egresados para la validación del proceso formativo, la generación de sistemas de gestión transparentes, eficaces y eficientes; la internacionalización, los procesos formativos flexibles e interdisciplinarios, el desarrollo de competencias y los recursos físicos y financieros adecuados y suficientes, entre otros.

Así es lógico concluir que «en la configuración de esos factores, pesan los recursos institucionales, los procesos internos y los resultados de la gestión académica» (CNA, 2013, p. 12) o dicho de otra manera, en el tránsito hacia la calidad de la educación, hoy deben tenerse en cuenta simultáneamente múltiples dispositivos que van más allá de lo concerniente a los componentes y los actores que directamente intervienen en el proceso docente educativo ${ }^{2}$.

Pero cada uno desde su lectura, su «verificación»y su «cumplimiento» en los ejercicios cotidianos dejan tras de sí una enorme estela que genera incertidumbre sobre lo que hay de «transversal» en todos ellos o lo que debe otorgarles el carácter de «complementariedad» pues como afirma Iafrancesco (2004b) «solo atinan a buscar evaluar infraestructuras locativas, recursos, contratos y convenios sin hacer el debido énfasis en los proyectos educativos y pedagógicos, desde los cuales se construye escuela y desde los cuales se da sentido, significado y estructura» (p. 10).

En consecuencia, la revisión de los factores y sus características no puede atender sólo a la provisión de bases de datos para posteriores decisiones que en su mayoría parecen consolidarse en torno a aspectos operativos y

2 Según Álvarez y González (2003) los componentes del proceso educativo son: el problema, los objetivos, los contenidos, el método, la forma, los medios y la evaluación.

ITINERARIO EDUCATIVO • ISSN OILI-Z753 • AÑO XXVII, N. ${ }^{\circ}$ 6I • ENERO - JUNIO DE ZOI3 • P. 2O9-2Z6 
de procedimientos, ya que «el fin de buscar la calidad en las universidades es establecer mecanismos para asegurar la pertinencia y pertenencia de los procesos de docencia, investigación y extensión, funciones centrales de la educación superior» (Tobón, Rial, Carretero y García, 2006, p. 65), lo que implica otros espacios de «desarrollo y transformación» generadores de dinámicas más formativas, académicas, integradoras y complejas para los procesos, que den vida a «nuevas culturas organizacionales y nuevas gestiones administrativas» (Iafrancesco, 2003, p. 42) y que nutran contextualizada y explícitamente el currículum.

\section{El curriculum como punto de convergencia}

El curriculum es precisamente el punto de encuentro y asimismo el eje dinamizador de todos los procesos llevados a cabo en la universidad para dar respuesta al designio de formación específico de los profesionales; pero la comprensión de tal afirmación se hace posible si se entienden mínimamente dos de los tres ${ }^{3}$ grandes enfoques (Grundy, 1998, p. 27) desde los cuales han tomado cuerpo sus definiciones:

\section{El curriculum como producto}

El punto de partida inicial, que se sustenta en una visión o perspectiva técnica, lo ilustran definiciones como las que cita Grundy (1998, p. 45) acerca del curriculum como producto:

Un conjunto interrelacionado de planes y experiencias que un estudiante lleva a cabo bajo la orientación de la escuela (Marsh y Stanfford, 1984, citado en Grundy, 1998, p. 3).

Todas las experiencias planeadas que la escuela prepara para ayudar a los alumnos a alcanzar los resultados del aprendizaje señalados y que corresponden a lo mejor de sus habilidades (Neagley y Evan, 1967, citado en Grundy, 1998).

Un programa de actividades (que han de realizar profesores y alumnos) diseñado de manera que los alumnos alcancen en la mayor medida posible determinados (Barrow, 1984, citado en Grundy, 1998, p. 1).

3 Grundy (1998) al aludir a la «teoría de los intereses constitutivos de Habbermas» como sustrato para conceptualizar el curriculum, presenta además el enfoque crítico». 
Entendido así, desde un enfoque técnico, el curriculum puede asimilarse a un programa o a un plan de estudios diseñado bajo un supuesto de predicción que «permite anticipar cómo será (probablemente) el ambiente futuro a partir de nuestra experiencia respecto de su forma actual» o cómo será el estudiante o producto al finalizar el proceso educativo, es decir dispone lo que debe saber el alumno o las destrezas con las que contará al final del proceso de formación; o en términos de las demandas actuales, prescribirá las competencias que le serán propias al graduarse.

Pero el problema no radica en estas disposiciones previas, ya que es totalmente válido y pertinente partir de unos supuestos predeterminados por ejemplo para la definición de un perfil profesional. El «talón de Aquiles» se ubica acá en la génesis de las mismas ya que es sólo la idea previa de los técnicos, del profesor o del personal administrativo adscrito al programa ${ }^{4}$ la que determina los contenidos - el «qué enseñar»- o los objetivos que darán cuenta de los productos a alcanzar. Una idea que hoy usualmente se fundamenta en la pregunta «qué debe saber el estudiante al concluir el ciclo».

En este contexto, el profesor tiene la responsabilidad en primera instancia de diseñar un plan; luego su tarea consistirá en ejecutarlo y ayudar a «reproducir» posteriormente en cada estudiante toda una serie de contenidos mediante su transmisión o un adiestramiento, con actividades o ejercicios, para el desarrollo de las destrezas.

Para organizarlos acude a estructuras lineales que se esbozan en las «materias» o «asignaturas» incluidas en un plan de estudios y entre las cuales sólo se derivan relaciones jerárquicas formalizadas en los «requisitos»y los «prerrequistos», lo que significa ausencia total de vínculos dialógicos, complementarios, inter y transdisciplinarios o complejos. Cada asignatura se erige sobre un cuerpo de contenidos, usualmente teóricos, que deben entenderse por sí mismos y que deben reproducirse más tarde de manera oral o escrita en una evaluación.

Hoy el tema de las asignaturas sigue vigente ya que por ejemplo, los Lineamientos para la Acreditación de Programas (CNA, 2013, p. 29) las

4 Anteriormente, y de manera particular en el caso de la educación básica y la media en Colombia, las «ideas previas» provenían de los técnicos o de los expertos (diseñadores) nombrados por el estado. El profesor en estos casos era sólo un ejecutor.

ITINERARIO EDUCATIVO • ISSN OIZI-2753 • AÑO XXVII, N. ${ }^{\circ}$ 6I • ENERO - JUNIO DE ZOI3 • P. ZO9-Z26 
tratan puntualmente (ver característica 16). Al respecto, y sólo de soslayo, para no vulnerar la finalidad de este artículo, sería interesante develar la intención de dicho texto al incluirlas de manera simultánea en propuestas de formación para las que igualmente se exigen competencias, ya que es difícil pensar un diseño curricular asignaturista que armonice con una orientación hacia la formación de las mismas.

En síntesis y para aportar al objeto de este escrito, vale enfatizar acá en el lugar del curriculum como producto que se materializa en un plan de estudios o un programa que emerge de la idea de un individuo o de unos pocos, usualmente el profesor y/ o los administradores académicos, y que por lo tanto carece de la participación, en su construcción y en su puesta en acción de otros actores y otros elementos de índole institucional.

\section{El curriculum como praxis}

Adentrarse hora en una concepción diferente del curriculum exige cambiar los «anteojos» (Khun, 1992, p. 38) conceptuales con los que se mire la realidad educativa para entender desde otro paradigma el fenómeno curricular de una manera incluso opuesta a un enfoque técnico.

Como «construcción social que forma parte de la estructura vital de una sociedad»(Grundy, 1998, p. 27), de sus dinámicas, sus valores, sus significaciones, los sentidos y concepciones sobre el mundo y los sujetos, el curriculum implica acción, dinamismo, interacción. En coherencia con esto, deja de ser un producto estático, para constituirse en proceso en el que intervienen distintos actores, «esto significa que hemos de buscar el curriculum, no en la estantería del profesor sino en las acciones de las personas inmersas en la educación» (Grundy, 1998, pág. 21), por eso le son inherentes todas las «prácticas educativas» de una institución.

Darle vida entonces, conocerlo, apropiarlo, evaluarlo invita en términos de Lundgren (1997), citado por Sacristán y Pérez (1992, p. 148), mirar sus contextos de formulación y de realización; y ambos trascienden el espacio y las posibilidades del profesor, para determinarse simultáneamente en las dinámicas, decisiones, pautas de acción y fenómenos generados por las múltiples instancias y por elementos que impactan el acontecer educativo. 
Definirlo es describir las funciones de la propia escuela ${ }^{5}$ y la forma particular de enfocarlas en un momento histórico y social determinado, para un nivel o modalidad de educación, en un entramado institucional (Sacristán G. , 1995, p. 16); así su mirada hoy en la esfera universitaria nace, se orienta y se nutre desde la docencia pero compromete decisivamente a la investigación, la extensión o proyección social.

Ahora bien, las funciones que el curriculum realiza según Sacristán (1995, pág. 65) tienen que ver con los contenidos, pero se circunscriben incluso a los «códigos pedagógicos» y a acciones prácticas a través de las que se expresan y moldean contenidos y formas.

En lo concerniente a los primeros, y si es cierto que el curriculum permite el acceso al conocimiento, no se trata sólo del conocimiento académico o intelectual, porque los contenidos son asimismo derivados de las interacciones, los significados y los sentidos que el sujeto construye en su relación con el mundo, es decir son culturales; y son también formativos, bien sea que la escuela los entregue de manera formal e informal, tácita o expresa; de ahí que

es una praxis antes que un objeto estático emanado de un modelo coherente de pensar la educación o los aprendizajes de los niños y de los jóvenes, que tampoco se agota en la parte explícita del proyecto de socialización cultural de las escuelas (Sacristán, 1995, p. 16).

Por consiguiente, los códigos se infieren hoy, en primera instancia, del Proyecto Educativo Institucional, y determinan el hacer que posibilitará llevar a cabo una propuesta de formación hasta que el estudiante culmine su proceso de aprendizaje en el marco de un perfil determinado. Él dota de «sentido e intencionalidad la acción educativa» (López Jiménez, 1999, p. 54) ya que plasma los principios «Antropológicos, axiológicos, formativos, científicos, epistemológicos, metodológicos, sociológicos, psicopedagógicos, didácticos, administrativos y evaluativos que inspiran los propósitos y procesos de la formación integral» (Iafrancesco , 2004a, p. 26) De esta manera aspectos como el modelo o el enfoque pedagógico elegido por la universidad, sus principio, sus concepciones sobre la ciencia

5 Se entiende la escuela como espacio físico y simbólico en el que se generan múltiples interacciones con el propósito de orientar y promover la formación integral de los sujetos inscritos en cualquier nivel educativo formal. 
y el conocimiento, su propia autoimagen como empresa o como centro para la generación o transmisión de conocimientos, la organización que se formaliza en las mallas curriculares, los espacios y tiempos, el lugar del docente y del estudiante en los procesos investigativos y de proyección social, las decisiones administrativas y muchos otros, considerados por Lundgren como «textos de representación» (Kemmis, 1998, p. 39) inciden en la «ordenación, secuencia, instrumentación metodológica y presentación de los curricula ${ }^{6}$ a los profesores y los alumnos» .

Pero de igual manera las «acciones prácticas» no sólo de los profesores, sino incluso de otro tipo de personal de la escuela, hacen posible tal realización, bien sea porque se llevan a cabo deliberadamente y con intencionalidades concretas o porque se asumen y hacen realidad desde su oscurecimiento u olvido Lundgren, 1997 en el curriculum oculto que plantea Lundgren (Sacristán G. , 1995, p. 108).

Los centros de formación ejercen su influencia formativa más allá de lo que son las actividades programadas tanto formales como informales. El propio hecho de asistir a una institución y la forma en que allí se trabaja ejerce una influencia evidente en los alumnos/as. El tipo de relación entre profesores y alumnos, los códigos de disciplina, el clima de trabajo, la tradición de esa institución, las cosas a las que se da más importancia, las expectativas que se proyectan sobre los estudiantes, etc. (Zabalza, 2007, p. 33).

En este sentido autores como Iafranfrancesco (2004a, p. 50) determinan «los medios» que posibilitan «la formación integral de los educandos» como parte fundamental del curriculum. De esta manera no sólo aquellos que previamente se definen e instauran para tal fin, sino incluso otros menos explícitos, son decisivos: «la gestión estratégica y estructura organizacional escolar...los contextos endógenos y exógenos situacionales, los recursos locativos-materiales-instrumentales...»

Por lo tanto asumir el curriculum como praxis lleva consigo implícita una disposición permanente de la universidad, en nuestro caso, y no sólo de los docentes o sus colectivos, a la autocrítica y la autorreflexión para dar luz a nuevas posibilidades de interpretación de la realidad que den cabida

6 La expresión «curricula» es el plural de la expresión latina «curriculum» que a su vez significa camino, carrera, trayecto. 
a múltiples oportunidades de resignificación de las prescripciones, los planteamientos pedagógicos y didácticos, las prácticas, la cultura, las metodologías, los procesos, las evaluaciones y que paralelamente favorezcan espacios innovadores pertinentes despojados del sello tan usual del activismo derivado del afán por tener productos que mostrar o tareas que entregar.

En este orden de ideas, es igualmente fundamental develar la incidencia de instancias en los que se forjan influencias explícitas e implícitas para la formación de los profesionales, así también estudiantes, pero del mismo modo los actores adscritos a los subsistemas político administrativo, académico, de producción de medios, de bienestar, de egresados, entre otros, son fundamentales y deben convocarse a una participación crítica y activa.

Como «espacio de toma de decisiones formativas» (Zabalza, 2007, p. 14) en el que se «entrecruzan diversas dimensiones» la universidad está llamada a una permanente toma de conciencia sobre las expectativas que en ella tiene puestas la sociedad sobre su marco formativo que se traduce en cada perfil de formación y sobre su misión particular con el mejoramiento de las condiciones y la transformación de la sociedad; por tanto mirarse desde modelos como el del CNA, u otro, es una responsabilidad que rebasa los límites de los formalismos y las ejecuciones para dar cumplimiento a un deber y a una obligación centrados en la garantía de los derechos individuales de los sujetos que en consecuencia se hace operativa, en primera instancia, en el currículo como «proyecto formativo que se pretende llevar a cabo en una institución formativa» (Zabalza, 2007, p. 21).

Esto implica que su interés en el currículo debe ser una apuesta permanente como evidencia incluso de un esfuerzo denodado y de una clara postura política, por evitar «bajos resultados» $\mathrm{y}$ «baja calidad», pero al mismo tiempo, las exclusiones sociales, en las que los currículos tienen una alta incidencia (Estebaranz García, 1999, p. 189).

\section{El curriculum: antes que las competencias y los factores de autoevaluación}

Varios interrogantes toman forma desde los planteamientos anteriores: ¿Debe inscribirse la formación por competencias en una propuesta del curriculum como producto o por el contario es necesario que su génesis se instaure en un curriculum como praxis? ¿Es pertinente hablar de autoevaluación para la calidad en el contexto del curriculum como 
producto? ¿Tienen cabida simultáneamente la autoevaluación para acreditación y la formación por competencias en un contexto de calidad mediado por el tema del curriculum?

Seguramente para muchos técnicos estará claro que los tópicos acá bosquejados pueden pensarse como un algo en solitario, único o fragmentado, pero más allá de tratarlos como requisitos independientes hacen parte del currículo y ambos a su vez lo impactan y constituyen; pero igualmente es éste el que les otorga sentido y permite concebirlos más allá de tareas por cumplir en el espacio de los legalismos, con lo que será viable dejar fuera de lugar inquietudes como las que manifiesta Torres (1994, p. 16):

\begin{abstract}
Muchas veces suele darse el caso de que, para estar a la moda o cumplir la legalidad, se muda sólo la apariencia de las propuestas, pero en el fondo, se sigue haciendo lo mismo. La rica filosofía de conceptos como los que estamos hablando, puede acabar en mera rutina, en propuestas tecnificadas, completamente ajenas a los problemas que sirvieron de estímulo para su formación.
\end{abstract}

Pero las implicaciones para superarlo exigen a la universidad repensarse y forjar un «debate interpretativo que permita reconocer posturas teóricas definidas frente la función y misión de la universidad» (Bentonilla, Pedranzani y Clavijo, 2007, p. 23) y que en consecuencia demarcarán rutas para entender que los perfiles de formación y el currículo que da cuenta de su configuración es un encargo de alta envergadura del cual no son sólo depositarios algunos docentes o «empleados».

Entonces tendrán que demarcarse nuevos retos orientados a «encontrar otras formas de conocimiento escolar, rescatar el sentido de la formación, revisar la racionalidad asentada en la llamada alta cultura» y «pujar por el resurgimiento de otros valores, de otros conocimientos, de otra idea de calidad de vida y de bienestar» (Sacristán, Rafael , Perrenoud, y Linuesa, 2011, p. 46).

Seguramente después de estas conquistas habrá grandes transformaciones:

Las universidades tendrán nuevos insumos para nutrir cultura organizacional (Castañeda y Fernández, 2007, p. 3) con el aprendizaje integral y sistemático sobre esos aspectos, así no sólo los docentes 
sabrán respecto al currículo y algunos administradores respecto a la autoevaluación y la acreditación. De esta manera se constituirá una cultura menos centrada en la tarea y los procedimientos, y más en la formación integral.

Desde seta óptica, los niveles de aprendizaje de los equipos directivos también serán impactados por cuanto se situarán más allá de aprender a trabajar con el resto de la comunidad educativa como lo devela la investigación (Ahumada Figueroa, Galdames y González, 2009, p. 354) y estarán motivados y convencidos sobre el impacto de su gestión en la calidad e integralidad del currículo, en la labor pedagógica y en la formación integral de los estudiantes en general.

De igual manera la vida propia de la universidad trascenderá los ámbitos técnico reproduccionistas y eficientistas, y se vislumbrará como un «espacio ecológico» en el que continuamente se produce, en términos de Torres (1991, p. 114) algo diferente a lo que está previsto y aconseja el modelo oficial»y y el espacio de lo legítimo en cuanto a las funciones de la universidad compete.

Respecto a los elementos que se evalúan y sus criterios de revisión, por ejemplo los factores que presenta el modelo de autoevaluación del CNA, serán algo más que los encabezados de unas listas de chequeo, o los subtítulos de los planes de mejoramiento, y se mirarán como dispositivos complementarios y convergentes en el curriculum como praxis trascendental en la formación de los sujetos que cada semestre deben entregarse a la sociedad con una impronta que los distinga por su integralidad, sus competencias laborales y su capacidad de responsabilidad social.

Finalmente quienes estamos llamados a promover una educación de calidad, podremos superar el temor a vivir un retroceso que implicaría la «redefinición del curriculum en los términos pragmáticos e instrumentales» (Giroux, 2004, p. 68) y lograríamos en un esfuerzo mancomunado «resaltar la relevancia del curriculum en los estudios pedagógicos, en la discusión sobre la educación y en el debate sobre la calidad de la enseñanza» para «recuperar la conciencia del valor cultural de la escuela como institución facilitadora de cultura, que reclama inexorablemente el descubrir los mecanismos a través de los que cumple dicha función y analizar el contenido y sentido de la misma» (Sacristán, 1995, p. 20). 
De igual manera seríamos legítimos en el esfuerzo por poner en acción, por superar o por dejar de lado los requisitos técnicos y políticos derivados de contextos externos, ya que asumiríamos directamente la responsabilidad de determinar si las reformas educativas a cargo de la universidad asumen la educación como instrumento de cambio o como forma de adaptación (Iafrancesco, 2003, p. 56) y en consecuencia, si las mismas, promueven las exclusiones sociales que los curricula ${ }^{7}$ gestan, a veces sin predeterminarlo; o si por el contrario se constituyen en vectores para la construcción sociedades democráticas y equitativas en las que el desarrollo humano se constituye en prioridad vital.

\section{Referencias}

Ahumada Figueroa , L., Galdames, S., González, Á., \& Herrera, P. (2009). El funcionamiento del equipo directivo durante un proceso de autoevaluación institucional en el marco de políticas de aseguramiento de la calidad de la getión escolar en Chile. Universitas Psychologica, pp. 353-369.

Altermann, N. (2008). La construcción del curriculum escolar claves de lectura de diseños y prácticas. Recuperado el Marzo de 2013, de Ebsco Host: http://web.ebscohost.com/ehost/detail?sid=d20829e4-de8e4615-ad16-667b7a0a9c19\%40sessionmgr10\&vid=9\&hid=24\&bdata $=$ Jmxhbmc9ZXMmc210ZT1laG9zdC1saXZl\#db=zbh\&AN=67615073

Álvarez, C., y González, E. (2003). Lecciones de didáctica general. Medellín: Magisterio.

Bentonilla, S., Pedranzani, B., \& Clavijo, M. (2007). El campo de la formación universitaria: rasgos y contornos de los cambios del curriculum en un contexto de crisis estructural. Recuperado el Marzo de 2013 de Ebsco Host.

Cabrera , K., y González, L. (2006). Currículo universiario basado en competencias. Barranquilla: Uninorte.

Castañeda, D. y Fernández Ríos , M. (2007). Validación de una escala de niveles y condiciones de aprendizaje organizacional. Univ. Psychol, pp. 245-251.

CNA (2006). Lineamientos para la acreditación de programas. Bogotá: CNA.

7 Plural en latín para el término «curriculum». 
CNA (2013). Lineamientos para la acreditación de programas de pregrado. Bogotá: CNA.

Correa, C. (2004). Gestión y evaluación de la calidad de la educación. Bogotá: Magisterio.

Estebaranz García , A. (1999). Didáctica de innovación curricular . Sevilla: Universidad de Sevilla.

Giroux, H. (2004). Teoría y resistencia en educación. Argentina: Siglo XXI. Grundy, S. (1998). Producto o praxis del currículum. Madrid: Morata.

Iafrancesco, G. (2004a). Currículo y plan de estudios. Bogotá: Magisterio.

Iafrancesco, G. (2003). Cambios en educación. Perspectiva etnometodológica. Bogotá: Magisterio.

Iafrancesco, G. (2004b). Acreditación de los centros educativos. Autoevaluación y autorregulación. Bogotá: Magisterio.

Kemmis, S. (1998). El curriculum: más allá de la teoría de la reproducción. Madrid: Morata.

Khun, T. (1992). La estructura de las revoluciones científicas. Bogotá: Fondo de Cultura Económica.

López Jiménez, N. (1999). Retos para la construcción curricular: de la certeza al paradigma de la incertidumbre creativa. Bogotá: Magisterio.

Lundgren, U. (1997). Teoría del currículum y escolarización. Madrid: Morata.

Proyecto Tuning. (2007). Tuning América Latina. Reflexiones y perspectivas de la educación superior en América Latina. Barcelona: Universidad de Deusto.

Sacristán, G. (1995). El currículo una reflexión sobre la práctica. Madrid: Morata.

Sacristán, J. y Pérez Gómez, A. (1992). Comprender y transformar la enseñanza. Madrid: Morata.

Sacristán, J., Rafael , A., Perrenoud, P. y Linuesa , M. (2011 ). Diseño, desarrollo e innovación del currículum. Madrid: Morata. 
Tobón, S., Rial, A., Carretero, M. y García, J. (2006). Competencia, calidad y educación superior. Bogotá: Magisterio.

Torres , J. (1991). El curriculum oculto. Madrid: Morata.

Torres, J. (1994). Globalización e interdisciplinariedad: el curriculum integrado. Madrid: Morata.

Unesco (1998). Declaración mundial sobre la educación superior en el siglo XXI: visión y acción. París: UNESCO.

Unesco (2009). Conferencia Mundial sobre la Educación Superior - 2009: La nueva dinámica de la educación superior y la investigación para el cambio social y el desarollo. París: UNESCO.

Zabalza, M. (2007). Competencias docentes del profesorado universitario. Calidad y dearrollo. Madrid: Narcea. 